\title{
Sintonizando con la generación digital. La expansión de los organismos de juventud a las plataformas 2.0
}

\author{
Xabier MARTÍnEZ RoLÁN \\ Universidade de Vigo \\ Xabier.rolan@uvigo.es. \\ Teresa PiñeIRo-Otero \\ Universidade da Coruña \\ Teresa.pineiro@udc.es
}

\begin{abstract}
Resumen:
El intenso proceso de la digitalización vivido por la sociedad española ha alumbrado el nacimiento de la primera generación de jóvenes digitales. Esta generación debe interactuar con unos sujetos y estructuras analógicos, lo que pone de relevancia la existencia de divergencias entre sus esferas comunicativas.

La adaptación de ambas esferas constituye un imperativo para las instituciones con competencias en políticas de juventud, organismos que tienen en los nativos digitales su segmento prioritario.

El presente artículo efectúa una aproximación a los canales 2.0 que las entidades autonómicas de juventud utilizan para comunicarse con los jóvenes digitales para determinar la correspondencia existente.
\end{abstract}

Palabras clave: Nativos digitales; políticas de juventud; administración autonómica; comunicación digital; plataformas 2.0.

\section{Tuning into the digital generation. The expansion of youth organizations to the platforms 2.0}

\begin{abstract}
:
The intense process of digitalizing of Spanish society has risen up the first generation of digital natives. This generation interacts with analogical subjects and structures, what reveals the existence of divergences between their communicative spheres.

The adaptation of both spheres it's a "must" for the institutions which develops youth policies, institutions that aim digital natives as their gold target.

Present article it's an approach to social media channels of regional institutions which develops youth policies - entities that communicates with that digital youth, in order to determine their relationship.
\end{abstract}

Key Words: Digital natives; youth policy; regional administration; digital communication; social media.

\section{Referencia normalizada:}

Martínez Rolán, X. y Piñeiro Otero, T. (2014): Sintonizando con las generación digital. La expansión de los organismos de juventud a las plataformas 2.0. Historia y Comunicación Social. Vol. 19. Núm. Especial Enero. Págs. 265-278.-

Sumario: 1. Introducción. 2. Esbozando un retrato de la generación digital. 3. Internet, un espacio para la comunicación. 4. Metodología. 5. Resultados. 5.1. Redes Sociales. 5. 2. Consumo en movilidad. 5.3. Actualización y acceso a la información. 6. Conclusiones. 7. Bibliografía. 


\section{Introducción}

"La juventud no es más que una palabra". Con esta expresión, Bordieu (1990) puso de manifiesto el carácter de constructo sociocultural del término joven y su variabilidad de unas sociedades a otras. Por ejemplo, mientras Organización Mundial de Salud se refiere con este término al grupo etario comprendido entre los 10 y los 24 años (OMS, 1986:11), la Unión Europea en su EU Strategy for Youth (2009) integra en esta denominación a las personas de entre 15 a 29 años y en España determinadas líneas de actuación para jóvenes son de aplicación hasta los 34 años.

La integración de sujetos mayores de treinta años en la consideración de joven como resultado de la dilación de los ciclos vitales de los jóvenes españoles resultaría impensable en los años 80. En este década, con la creación del Instituto de la Juventud (INJUVE) se inició la creación de entidades autonómicas y locales destinadas a promover "las condiciones para la participación libre y eficaz de la juventud en el desarrollo político, social, económico y cultural" (Constitución Española, 1978, Título 1, art. 48).

José Manuel Pantón y Casas (en Montiel Roig, 2009) se refiere a un concepto administrativo de juventud, que toma como base una serie de ideas de carácter sociológico sumadas a las inercias de acción en la Administración que influyen en el modelo de intervención y en su objeto.

En este sentido, la ampliación en el tramo de edades que abarca el término "joven" en España pretende dar respuesta a las problemáticas concretas de la juventud, en su mayoría derivadas de un retraso en la emancipación de los jóvenes (Muñoz, 2006).

El amplio grupo etario que conforma la juventud española, desde una perspectiva administrativa, hace preciso un proceso de segmentación en la aplicación de las políticas para jóvenes. Sin llegar a profundizar en el concepto bourdieuano de la edad social y su ruptura con la concepción de la juventud como un grupo compacto y homogéneo, lo cierto es que un individuo de 15 años difícilmente tendrá las mismas demandas, reivindicaciones o necesidades que uno de 30.

El desarrollo de la Sociedad de la Información en España ha alumbrado a la primera generación digital. Los primeros ciudadanos españoles que han tenido contacto con Internet y las nuevas tecnologías desde su socialización primaria, los nativos digitales (Prensky, 2001: 1) se ha hecho mayor y ya se corresponden con la franja más joven de la juventud española.

Esta generación piensa y procesa la información de un modo completamente diferente a sus predecesores, que constituyen inmigrantes tecnológicos, lo que implica la existencia de una brecha digital etaria -incluso- entre individuos generacionalmente próximos.

Frente a los jóvenes inmigrantes digitales, cuyo contacto con las TICs se produjo en su infancia o adolescencia, los nativos digitales han pasado toda su vida rodeados 
de equipamiento tecnológico convirtiéndose en usuarios tempranos de ordenadores, videojuegos, reproductores multimedia, videocámaras, etc.

En su mayoría, los jóvenes digitales han tenido acceso frecuente a un ordenador en la infancia y su primer teléfono móvil en la preadolescencia. Esta cotidianidad con la tecnología ha afectado a la percepción de ésta, ya no ven equipamiento tecnológico sino máquinas para la comunicación (Boschma, 2006).

Los elevados índices de conexión y uso de las nuevas tecnologías entre los nativos digitales han sido considerados uno de los rasgos definitorios de esta generación (2013:13). Inclusive en el plano neurobiológico, la continua interacción con la tecnología ha llevado a estos jóvenes a reaccionar cada vez más de prisa y codificar la información de forma diferente (Small citado en Matellanes, 2011: 12).

Asimismo, estos nativos digitales tienen una gran capacidad de comunicación y una necesidad de expresarse mayor que los inmigrantes digitales, lo que va a afectar a su interacción con sujetos y entidades al tiempo y les lleva a asumir roles activos en el acceso y consumo de la información.

\section{Esbozando un retrato de la generación digital}

El desarrollo y penetración de las TICs en los hogares españoles ha logrado situar a España entre los países del mundo con mayor inclusión digital.

Hoy, el número de internautas supera los 24,1 millones de personas; esto es, más de la mitad de la población española de entre 16 y 74 años ha accedido a la Red en los últimos tres meses de los cuáles el 72,6\% se conectan a diario (Fundación Telefónica, 2013).

A pesar de que el avance de la Sociedad de la Información en España ha logrado minimizar las diferencias en el alcance y adopción de las nuevas tecnologías todavía resulta preciso hablar de una brecha digital etaria particularmente patente entre los nativos digitales y las generaciones precedentes.

La edad constituye un factor relevante en lo que respecta a la adopción y apropiación de las nuevas tecnologías; de hecho cuatro de cada diez internautas españoles son menores de 35 años, la mayoría de los cuáles pertenecen a la generación de nativos digitales (ONTSI, 2013). La generación digital también concentra el mayor volumen de internautas intensivos (un $95,5 \%$ frente al $87 \%$ de los jóvenes de entre 25 a 35 años, ONTSI, 2013).

Sin embargo es en el ámbito de la llamada "cultura de la portabilidad" (Kischinhevsky, 2009) donde los nativos digitales han desarrollado sus dominios.

La penetración de la telefonía móvil entre los jóvenes digitales alcanza prácticamente a todos los individuos de entre 16 a 24 años; de los cuáles un $68,3 \%$ ya cuenta con un terminal inteligente entre su equipamiento personal (27 puntos por encima 
que la penetración total de los smarphones en España, ONSI, 2013; Fundación Telefónica, 2013).

La rápida adopción de los terminales inteligentes así como de otros dispositivos portátiles de altas prestaciones ha potenciado la eclosión de nuevas utilidades basadas en la tecnología de dichos terminales y en las conexiones de datos inalámbricas. La incorporación de estas utilidades asociadas a la movilidad ha conllevado el desarrollo de nuevas tendencias de acceso y consumo de los contenidos, en los que la generación digital ha asumido el papel de motor de cambio.

Son los internautas españoles 16 a 24 años quiénes encabezan el acceso a Internet desde dispositivos móviles. El 66,7\% de los jóvenes digitales acceden a la Red desde el teléfono móvil y el $51 \%$ desde el ordenador portátil, aventajando en veinte puntos porcentuales al conjunto de internautas españoles (INE 2012)

El incremento de las conexiones en movilidad lleva implícito el acceso a Internet varias veces al día, lo que confiere una nueva dimensión al uso intensivo de la Red. En este sentido resulta preciso hacer referencia a una conexión continua que ha dado lugar a la aparición de un usuario permanentemente conectado, situación en la que se encuentran cuatro de cada diez internautas de entre 16 y 24 años (Telefónica, 2013). Este usuario cuyo acceso y consumo de contenidos responde al paradigma de always $\&$ everywhere \& anydevice, ha logrado rescatar para el ocio microtiempos cotidianos anteriormente perdidos.

La conexión permanente ha estimulado la necesidad voraz de información de los nativos digitales. Se trata de una generación acostumbrada a recibir información de forma rápida durante los múltiples accesos que efectúa diariamente a la Red. El consumo en microaccesos ha repercutido en el tamaño y estructura de los contenidos, cada vez más breves para una lectura rápida aunque con la posibilidad de seleccionar diversos niveles de profundidad.

También como resultado de esta conexión permanente, a la que es preciso sumar el incremento en la capacidad de almacenaje de los terminales tecnológicos, los nativos digitales han efectuado una "delegación de la memoria". Más que recordar datos, estos jóvenes han desarrollado competencias de búsqueda y selección de la información en un proceso que, tomando como referencia a Vaidhyanathan, podría denominarse de "googlización" del conocimiento (2011).

Otra característica de las rutinas del consumo online de los nativos digitales es su tendencia al multitasking. Con frecuencia estos jóvenes acceden a la vez a contenidos a través de diversos dispositivos, en diferentes lenguajes dada su capacidad de consumir información en paralelo. Como afirman Reig y Vílchez (2013: 27), más que a un incremento en la capacidad de recepción dentro de un entorno saturado de información, se debe hacer referencia a una habilidad para simultanear tareas que requieran diversos grados de atención. 
El consumo multitasking constituye, junto al acceso always \& everywhere \& anydevice, una potente influencia en la readaptación de contenidos para estos jóvenes digitales.

\section{Internet, un espacio para la comunicación}

Una vez analizadas las características de acceso y consumo de las nuevas tecnologías más representativas de la generación digital, resultó de interés determinar los usos más frecuentes que dan a la Red.

La utilización que los nativos digitales efectúan de Internet se corresponde con un patrón lúdico-social: interacción en las plataformas sociales, descargas de archivos audiovisuales, imágenes, juegos y distribución de contenidos de creación propia.

El uso estos jóvenes dan a los servicios online de ocio y comunicación supera al de la media internautas españoles en 10 puntos porcentuales en contenidos lúdicos (con un $48 \%$ respecto a un $39 \%$ y hasta 16 en el caso de los servicios de comunicación (con un 93\% respecto a un $80 \%$ ). La generación digital solamente se encuentra por debajo de la media española en el consumo de servicios ligados a la productividad (con un 38\% respecto a un 56\%, Fundación Telefónica, 2013).

Sin embargo es la comunicación con su entorno el motivo principal que alegan los nativos digitales para su uso de Internet. Una comunicación que ha vivido un giro radical con la irrupción de servicios de mensajería instantánea estilo WhatsApp, que ya se erige como el tercer modo de comunicación entre los jóvenes (después del contacto cara a cara y el móvil) así como de las redes sociales (que ocupan cuarto lugar en modos de comunicación).

Precisamente es la conexión con el entorno la que constituye la principal motivación para el uso de las redes sociales. El 92\% de los usuarios de Tuenti, el 82\% e Facebook y el $69 \%$ de Twitter utilizan estas plataformas sociales con una finalidad comunicativa (Fundación Telefónica, 2013). De hecho, a pesar de las posibilidades de creación de contenidos propios que brinda el entorno digital, los internautas españoles suelen utilizar las redes sociales para el envío de mensajes privados, el seguimiento de las actualizaciones de sus contactos o el chat.

Dice Caldevilla (2010) que en la era de las Redes Sociales la pulsión humana de compartir experiencias, saberes, vivencias u opiniones puede alcanzar su culmen. En el caso concreto de los nativos digitales además, se trata de una generación que funciona mejor conectada.

Esta particularidad que ya había señalado Prensky en su aproximación a la nueva generación nacida en un entorno sociodigital, tiene su máximo exponente en las redes sociales. 
Los nativos digitales se han apropiado de las redes sociales convirtiéndolas en "un espacio social y personal de su actividad, de su forma de actuar, de relacionarse con el mundo y con otras personas" (García García y Rosado Millán, 2012: 16). Si en el contexto español la penetración de las redes sociales es de un $63 \%$ este porcentaje se incrementa hasta el $85 \%$ en la franja de entre 14 a 24 años (Fundación Telefónica, 2013), aunque con divergencias en el acceso y uso de estas plataformas sociales.

Gráfico 1. Penetración de RR.SS. entre los internautas españoles.

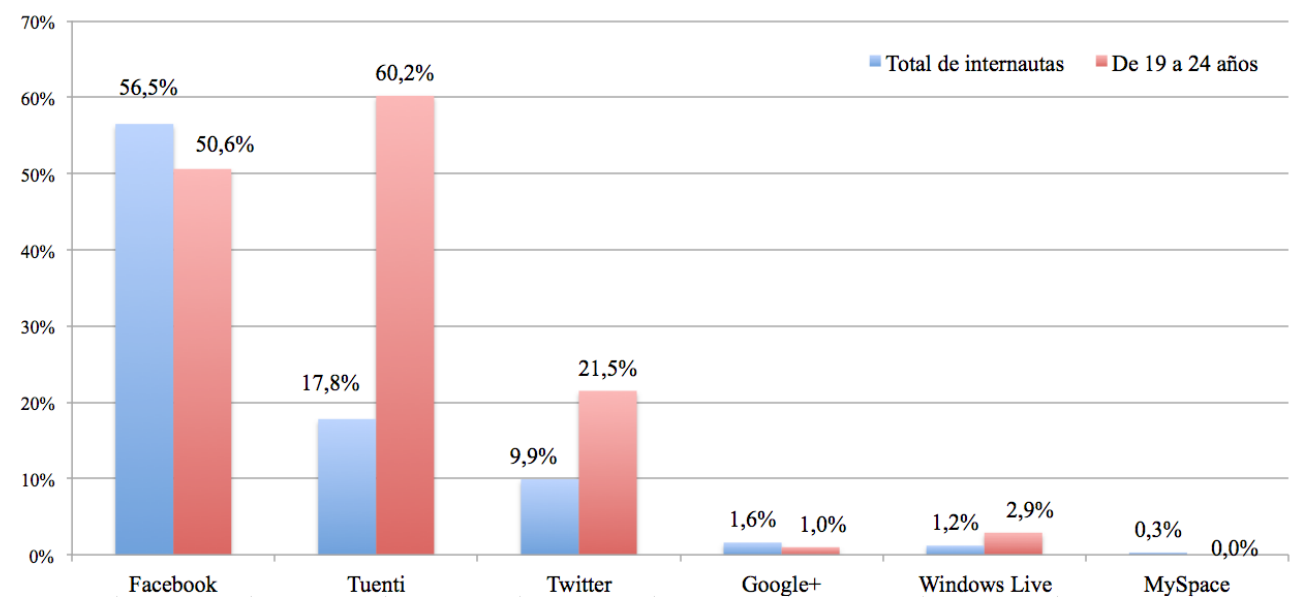

Elaboración propia a partir del Informe de la Sociedad de la Información en España 2013.

Como refleja el gráfico1, Tuenti es la red social joven por excelencia. El 60,2\% de los internautas de entre 19 a 24 años presenta un perfil activo en esta plataforma; presencia que desciende hasta el $17,8 \%$ si se atiende al total de usuarios de redes sociales.

Facebook, la plataforma con mayor número de usuarios activos en España, se sitúa en segundo lugar si se atiende a su penetración en la generación de nativos digitales. Esta menor presencia puede responder a la percepción de dicha red social como una plataforma de evolución; Tuenti constituye la primera plataforma entre los jóvenes de 14 a 17 años mientras que Facebook domina el segmento de los 18 a los 30 años.

En lo que respecta a Twitter resulta preciso destacar la expansión vivida por la red de microblogging en el último año, que tuvo especial incidencia entre los más jóvenes. Hoy, la penetración de esta plataforma social en la franja de internautas de entre 19 a 24 supera ya el $21 \%$, situándose más de once puntos porcentuales sobre la media española.

Además de estas redes sociales, resulta preciso destacar divergencias en el uso de otras plataformas sociales en función de la edad. Este es el caso del ya desaparecido Windows Live, cuya mayor presencia de nativos digitales se puede relacionar con 
su iniciación como internautas durante la era Hotmail-Messeger (servicios de correo electrónico y mensajería instantánea interrelacionados); o de Google+ y MySpace.

Además de las divergencias en la presencia de nativos e inmigrantes digitales en las diferentes plataformas sociales, también se pueden señalar patrones de acceso y consumo de las redes sociales.

Las características de esta generación digital en términos de hiperconectividad, apropiación de las tecnologías y tendencias de consumo de los contenidos hace preciso que los emisores clásicos revisen su papel en el proceso comunicativo en términos de una mayor eficacia.

No obstante, pese a la intensiva digitalización del contexto actual estos jóvenes nativos deben hacer frente a unos sujetos y estructuras dominados por una generación analógica.

La necesidad de una "traducción" que permita la correspondencia entre las esferas comunicativas de nativos e inmigrantes, jóvenes y entidades, se convierte en un imperativo para las instituciones con competencias en materia de juventud. En este sentido, el presente artículo analiza la presencia de los organismos de juventud en la esfera 2.0 para determinar la convergencia de sus estrategias comunicativas en la red con las características de los nativos digitales y sus tendencias de acceso y uso de la Red.

Un análisis que permitirá determinar si estas entidades están en consonancia con los jóvenes digitales o si, por el contrario, se mueven en esferas completamente diferentes.

\section{Metodología}

Para determinar el grado de consonancia de los organismos de juventud con los jóvenes digitales se ha desarrollado un análisis cuantitativo-cualitativo de los servicios que estas entidades ponen a disposición de sus públicos en la Red, canales y contenidos.

La aproximación efectuada a la generación digital ha permitido el establecimiento de una serie de ítems de estudio como la presencia en social media, la oferta de contenidos para un consumo en movilidad o sobre la actualización de la información y las posibilidades de acceso a ésta. Unos ítems que han permitido el análisis de la estrategia online de las entidades de juventud en la primera semana de septiembre de 2013.

El hecho de que el estudio se haya enfocado hacia la Administración Autonómica ha conllevado la acotación de la muestra a un total de 17 organismos encargados de la gestión de las políticas de juventud en las Comunidades Autónomas.

Empero esta selección no ha estado exenta de problemas. Desde la primera aproximación a la muestra de análisis se ha constado la existencia, en algunas comuni- 
dades autónomas, de duplicidades entre el organismo de juventud y la secretaría o servicio autonómico de quién depende administrativamente. En dichos casos se optó por la elección de aquella entidad cuya denominación o misión explicitase en mayor medida sus competencias en materia de juventud.

En definitiva, los organismos que conformaron la selección de estudio han sido: Dirección Xeral de Xuventude e Voluntariado (Galicia), Instituto Asturiano de la Juventud (Asturias), Servicio de la Juventud (Cantabria), Instituto de la Juventud (Castilla y León), Gazteaukera (Euskadi), Instituto Navarro de Deporte y Juventud (Navarra), Instituto Riojano de la Juventud (La Rioja), Instituto Aragonés de la Juventud (Aragón), Direcció General de Joventut (Cataluña), Institut Valencià de la Joventut. Generalitat Jove (Valencia), Centre Balear d'Informació i Documentació per a la Joventut (Baleares), Dirección General de Juventud y Deportes (Madrid), Dirección General de Prevención de Violencia de Género, Juventud, Protección Jurídica (Murcia), Dirección General de Empleo y Juventud (Castilla la Mancha), Instituto Andaluz de la Juventud (Andalucía), Instituto de la Juventud de Extremadura (Extremadura) y Dirección General de Juventud (Canarias).

Resulta preciso destacar el hecho de que, en algunas comunidades autónomas, determinadas políticas de juventud como el Carnet Joven han generado un universo online de tal envergadura que, incluso, ha llevado a eclipsar al propio organismos gestor. Una cuestión que ha tenido injerencia en algunas de las cuestiones analizadas.

\section{Resultados}

El estudio desarrollado ha permitido determinar la existencia de divergencias no sólo entre las esferas comunicativas de la generación digital y los organismos de juventud, sino entre la adopción de las herramientas 2.0 por parte de las entidades autonómicas.

\subsection{Redes sociales}

El impacto de las redes sociales entre los jóvenes ha propiciado el salto de los organismos de juventud hacia estas plataformas sociales. De hecho, como se puede observar en el gráfico 3 prácticamente todos los organismos autonómicos presentan una cuenta o perfil activo en Facebook y Twitter. Presencia que en el caso de Tuenti solamente es común a once entidades. 
Gráfico 2. Organismos de juventud en las Redes Sociales (presencia).

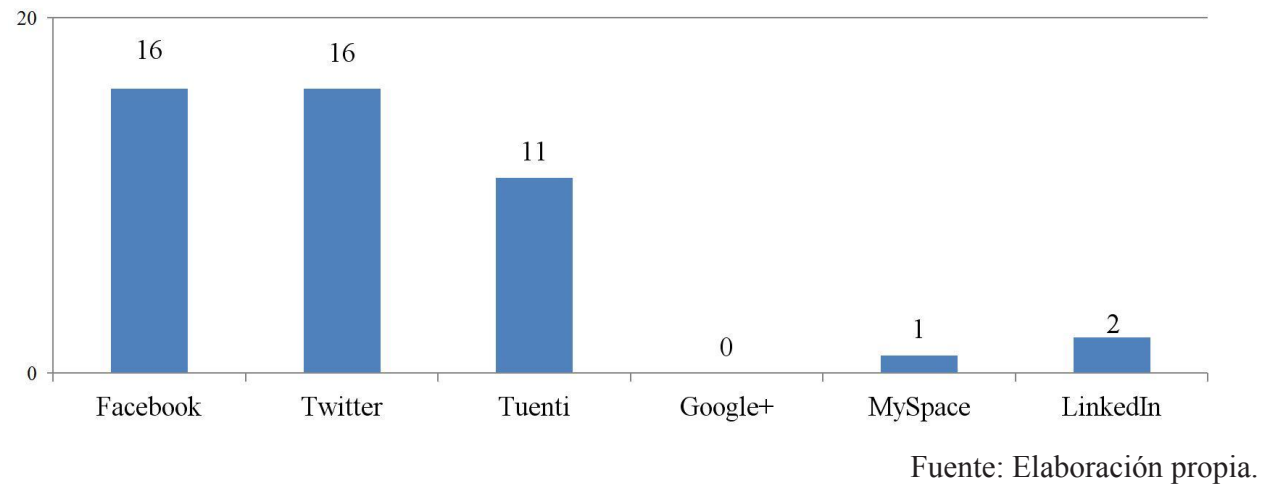

Algunos organismos de juventud han apostado por expandir su universo web a otras redes sociales aunque se trata de una tendencia que todavía resulta minoritaria (Gráfico 2).

En lo que respecta a su presencia en otras plataformas sociales (Gráfico 3), resulta destacable que trece de los organismos de juventud analizados cuentan con un canal en Youtube, y otros siete han hecho de Flickr su repositorio de fotografías particular.

Gráfico 3. Organismos de juventud en Social Media (presencia).

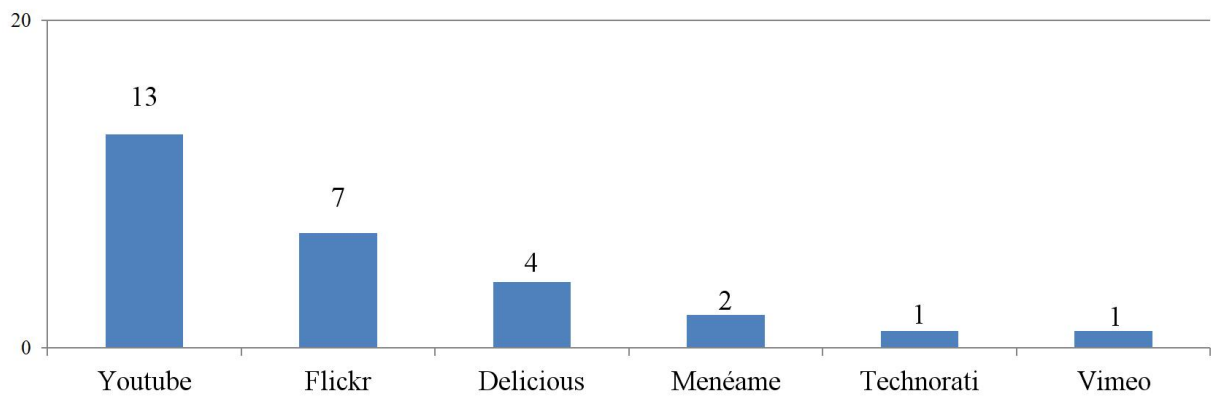

Fuente: Elaboración propia.

La presencia de los organismos de juventud en estas plataformas no sólo supone la utilización de un servicio de almacenamiento gratuito en la nube, sino que implica una mayor accesibilidad y compartibilidad de sus contenidos posibilitando una mayor difusión.

Esta necesidad de ampliar el alcance de su información y sus contenidos ha llevado a que cuatro de las entidades de juventud tengan un canal en plataformas como Delicious o Menéame (cuatro entidades y dos, respectivamente).

El análisis de la presencia de las entidades de juventud en las plataformas sociales ha permitido constatar una preferencia por aquellos social media con mayor impacto 
en la webesfera española general, más que en el ámbito de la juventud. En particular, esta preferencia implica una mayor visibilidad de la entidad autonómica en Internet, dado que son las redes sociales con mejor posicionamiento en el buscador Google, pero limitan las posibilidades comunicativas con la generación de nativos digitales.

Otra cuestión que resulta destacable, es el carácter 1.0 de la esfera comunicativa online de la Dirección General de Juventud y Deportes de Madrid. En el momento de realización del estudio constituía el único organismo autonómico sin presencia en las principales redes sociales; una ausencia que se suple con la utilización del foro como canal de interacción.

La carencia de una estrategia 2.0 del organismos gestor de las políticas de juventud madrileño contrasta con la entidad de una de sus principales líneas de actuación en la web. El Carnet Joven madrileño cuenta con perfiles activos en Facebook y Twitter, asumiendo la función informativa del organismo de juventud.

\subsection{Consumo en movilidad}

Frente al impacto de la cultura de la portabilidad en la generación de jóvenes digitales, los organismos con competencia en materia de juventud no han adaptado sus contenidos para su consumo a través de terminales móviles.

Solamente el 29,41\% de la muestra (un total de cinco entidades) cuentan con una página web adaptada para su acceso y consulta desde el teléfono móvil.

Oferta que, sin embargo, no garantiza un acceso optimizado a la web de dichos organismos desde dispositivos móviles. Si se atiende a determinados aspectos como el acceso y usabilidad de los contenidos, el número de entidades de juventud que ofertan sitios web adecuados para su consumo móvil desciende hasta el 17,65\% (un total de tres entidades).

El Instituto Asturiano de la Juventud y el Servicio de Juventud de Cantabria completan su oferta para el consumo en movilidad con la adaptación de su sitio web para tablets; una adaptación que tampoco se encuentra optimizada.

Si se atiende a las aplicaciones, sencillas extensiones de software para un acceso directo y fluido a los contenidos online, solamente la entidad de juventud de Castilla y León cuenta con una oferta de este tipo para terminales móviles iOs y Android.

En el caso de las APPs también se puede volver a señalar una transferencia de entidad del organismo gestor de juventud al Carnet Joven. Administraciones como la catalana y la gallega han creado una aplicación ex profeso para usuarios de dicha acción juvenil.

\subsection{Actualización y acceso a la información}

Los organismos de juventud deben aproximar sus tiempos y contenidos comunicativos clásicos a las nuevas tendencias de acceso continuo a la información de su público objetivo. En este sentido, además de su presencia en las redes sociales, se ha 
prestado atención a los restantes canales de información que dichas entidades ponen a disposición de la generación digital.

En el sitio web es el espacio "actualidad" (también denominado "noticias", "novedades", "lo último", etc.) el que cuenta con una mayor renovación de contenidos (en el caso de Mocedad Astur la actualidad se pasa al blog).

Gráfico 4: Frecuencia de actualización de contenidos de "Actualidad" en las web de los organismos de juventud.

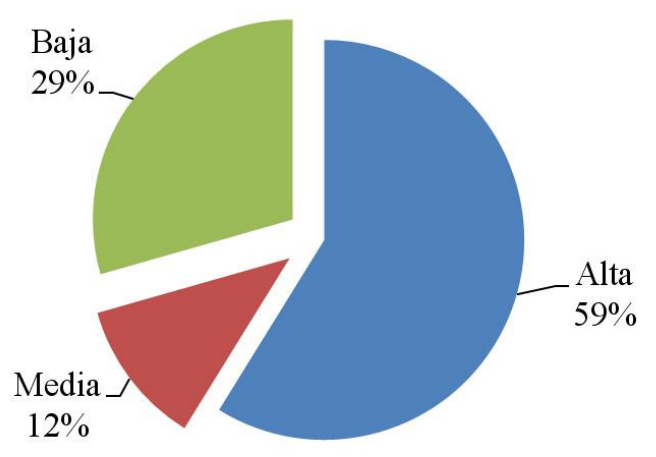

Fuente: Elaboración propia.

De prestar atención a la frecuencia de actualización de dicho apartado (gráfico 4) se puede señalar que la mayor parte de los organismos de la muestra publican información a diario e, inclusive, de forma continuada. Esta cuestión implica la adaptación de estas entidades a la tendencia de acceso y consumo de información de la generación de jóvenes permanentemente conectados.

Contrariamente, todavía persiste un porcentaje de organismos cuya frecuencia de actualización es semanal o, inclusive, inferior a ésta (frecuencias media y baja). La escasa renovación de contenidos informativos puede implicar una menor frecuencia de acceso de los usuarios a la web limitando su capacidad de engagement.

Para facilitar el acceso de la generación digital a estos contenidos informativos el $76 \%$ de las entidades analizadas han incorporado un sistema de sindicación de contenidos vía RSS o Atom.

Gracias a este servicio los usuarios suscritos puedan estar al día de las novedades que se produzcan en la página del organismo de juventud (en toda la página o en secciones concretas) de forma automática, sin tener que acceder al sitio web. En el Instituto Andaluz de la Juventud este servicio se complementa con otro de alertas SMS.

Otra vía de información que oferta el $65 \%$ de las entidades analizadas son los boletines. Herederos de las hojas información joven, estos boletines cuentan con diversos formatos en función de su grado de adaptación a Internet. 
El formato más frecuente, presente en el 30\% de las entidades analizadas, es el de un boletín tipo newsletter que permite a los usuarios suscritos en el sistema recibir cómodamente en su correo electrónico todas aquellas novedades vinculadas a las políticas de juventud de ámbito autonómico.

La adaptación del boletín a la web constituye un paso intermedio en el universo comunicativo web de estos organismos, dada la pérdida de retroalimentación presente en otras herramientas 2.0.

\section{Conclusiones}

El análisis de la adopción y apropiación de los medios sociales y otros servicios 2.0 por parte de los organismos autonómicos de juventud, ha permitido determinar diferencias en el engranaje de las esferas comunicativas online de entidades y públicos.

Con la evolución de la web (soportes, formato, accesibilidad, etc.) los sitios de los organismos de juventud se han completado con otros canales y servicios 2.0. Al mismo tiempo, han incorporado información cada vez más fragmentada y visual, en diversos formatos y lenguajes con una alta frecuencia de actualización.

Pese a estos cambios, el universo comunicativo online de los organismos de juventud no evoluciona con la misma rapidez que la adopción y apropiación de las TICs por parte de los nativos digitales, lo que hace compleja la convergencia de sus esferas comunicativas.

La apuesta por la expansión a las redes sociales de estas entidades, el salto a la segunda pantalla o -incluso- el sistema de acceso y distribución de la información se encuentra más próximo a la generación de inmigrantes digitales que a la de nativos digitales, lo que implica una desventaja comunicativa y la pérdida de engagement de los organismos de juventud entre una generación que vive en red y en la Red.

\section{Bibliografía}

BOSCHMA, J. (2006). Generación Einstein. Bilbao: Gestión 2000.

CALDEVILLA DOMÍNGUEZ, D. (2010). "El hombre binario". En Comunicación y Hombre, $\mathrm{n}^{\mathrm{o}}$ 6, p. 125-138.

COMISIÓN EUROPEA(CE) (2009). Una estrategia de la UE para la juventud: inversión y capacitación. Un método abierto de coordinación renovado para abordar los desafios y las oportunidades de los jóvenes. Luxemburgo. Oficina de Publicaciones Oficiales de las Comunidades Europeas. Disponible en http://eur-lex. europa.eu/LexUriServ/LexUriServ.do?uri=COM:2009:0200:FIN:ES:PDF [1-092013]. 
FUNDACIÓN TELEFÓNICA (2013). La Sociedad de la Información en España 2012. Barcelona: Ariel.

GARCÍA GARCÍA, F. y ROSADO MILLÁN, M.J. (2012). "Conductas sociocomunicativas de los nativos digitales y los jóvenes en la web 2.0". En Comunicación y Sociedad, vol. $25, \mathrm{n}^{\circ} 1$, p. 15-38.

IAB SPAIN y ELOGIA MEDIA (2013). IV Estudio anual Redes Sociales. Disponible en www.iabspain.net/redes-sociales [1-09-2013].

INE (2012). Encuesta de equipamiento y uso de tecnologías de la información y la comunicación en los hogares. Disponible en www.ine.es/prodyser/micro_tich. $\mathrm{htm}$ [[1-09-2013].

KISCHINHESKY, M. (2009). "Cultura da portabilidade. Novos usos do rádio e sociabilidades em mídia Sonora". En OBS Journal, nº 8, p. 223- 238.

MATELLANES LAZO, M. (2011). "Actitudes, comportamientos y usos de diferentes generaciones de usuarios en Internet" En: Revista Vivat Academia, $\mathrm{n}^{\circ} 115$. Disponible en http://www.ucm.es/info/vivataca/numeros/n115/DATOSS.htm [1-09-2013].

MONTIEL ROIG, G. (2009) "Transiciones virtuales en la juventud: una aproximación a la emancipación juvenil en la sociedad de la información". En: Recerca, revista de pensament $i$ análisi, $\mathrm{n}^{\circ}$ 9, p. 145-170. Disponible en http://www.e-revistes.uji.es/index.php/recerca/article/viewFile/152/139 [1-09-2013].

Observatorio Nacional de las Teclecomunicaciones y de la Sociedad de la Información (ONTSI) (2013). Informe Anual la Sociedad en Red 2012. Disponible en $\mathrm{http}: / /$ www.ontsi.red.es/ontsi/es/estudios-informes [1-09-2013].

ORGANIZACIÓN MUNDIAL DE LA SALUD (OMS) (1986). La salud de los jóvenes un desafio para la sociedad. Málaga: Gráficas Reunidas.

PRENSKY, M. (2001). "Digital Natives, digital Immigrants" En: On the Horizont, vol. $9 \mathrm{n}^{\mathrm{o}}$ 55. Disponible en http://www.emeraldinsight.com/journals.htm?articleid $=1532742$ [1-09-2013].

REIG, D. y VILCHES, L. F. (2013). Los jóvenes en la era de la hiperconectividad: tendencias, claves y miradas. Madrid: Fundación Telefónica y Fundación Encuentro.

VAIDHYANATHAN, S. (2011). The Googlization of everything. Berkeley: University of California Press.

\section{Los autores}

Xabier Martínez Rolán es Licenciado en Comunicación Audiovisual por la Universidade de Vigo, con Diploma de Estudios Avanzados en en el programa de Doctorado de Procesos Políticos Contemporáneos de la Universidad de Santiago de Compostela. Ha centrado su ámbito de investigación en las plataformas sociales y la comunicación digital, ámbito en el que también desarrolla su actividad profesional. En la actualidad es profesor asociado de la Facultad de Ciencias Sociales y de la Comunicación de la Universidad de Vigo. 
Teresa Piñeiro-Otero es Doctora por la Universidad de Vigo y Licenciada en Publicidad y Relaciones públicas por la misma universidad. Entre sus líneas de investigación destaca el estudio de nuevas fórmulas de comunicación online; una línea sobre la que ha publicado en diversas revistas nacionales e internacionales. En el momento actual es profesora de Estrategias de Comunicación Multimedia en el Grado de Comunicación Audiovisual de la Universidade da Coruña. 